\title{
Simulation realization of 2-D wavelength/time system utilizing MDW code for OCDMA system
}

\author{
M. S. A. Azura, C. B. M. Rashidi, S. A. Aljunid, R. Endut, and N. Ali \\ Advanced Communication Engineering, Centre of Excellence, School of Computer and Communication Engineering, \\ Universiti Malaysia Perlis, Kangar, 01000, Perlis, Malaysia.
}

\begin{abstract}
This paper presents a realization of Wavelength/Time (W/T) Two-Dimensional Modified Double Weight (2-D MDW) code for Optical Code Division Multiple Access (OCDMA) system based on Spectral Amplitude Coding (SAC) approach. The MDW code has the capability to suppress Phase-Induce Intensity Noise (PIIN) and minimizing the Multiple Access Interference (MAI) noises. At the permissible BER $10^{-9}$, the 2-D MDW (APD) had shown minimum effective received power $(P s r)=-71 \mathrm{dBm}$ that can be obtained at the receiver side as compared to 2-D MDW (PIN) only received $-61 \mathrm{dBm}$. The results show that 2-D MDW (APD) has better performance in achieving same BER with longer optical fiber length and with less received power (Psr). Also, the BER from the result shows that MDW code has the capability to suppress PIIN ad MAI.
\end{abstract}

\section{Introduction}

Optical Code Division Multiple Access (OCDMA) technology is one of the talented technologies to execute all-optical networks. It is a group of multiplexing and internetworking technologies that encodes/decodes signal through employing straightforward and cost-effective inactive optical component such as the signal multiplexing, routing and switching can be implement easily [1]. OCDMA can be realized in one-dimension (1D) and two-dimension (2-D) encoding through spatial/spectral domain. The execution assessment of OCDMA framework in view of wavelength/time (W/T) code has been investigated by measuring the estimations of bit error rates for various number of active users [2]. The network capacity of the OCDMA is in high cardinality, compatible code with various bandwidth demands, throughput and acceptable at $10^{-9}$-bit error rate (BER) [2, 3, 4]. The OCDMA systems undergo from assured noise such as phase induce-intensity noise (PIIN) shot noise and thermal noise [5]. Besides, multiple access interference (MAI) is the major performance degradation mainly when a large number of users are occupying in the OCDMA systems. Then, the most significant consideration is the code design for decreasing contribution at the MAI to the optical power receives [6]. In OCDMA systems, there are several techniques can be implemented to eliminate MAI. Spectral Amplitude Coding (SAC) is the one that can be used in this system. SAC schemes activate at bit rate, and as a result the condition for receiver bandwidth is tranquil $[7,8]$. Here, the performance of 2-D MDW codes for OCDMA systems to achieve higher performance possible through

\footnotetext{
${ }^{*}$ Corresponding author: amyazura92@gmail.com
}

suppressing PIIN and decreasing the MAI was analyzed $[9,10]$.

\section{Development of 2-D Modified Double Weight (MDW) code}

2-D MDW codes are also signified by using the $M \times$ $N$ matrix form. The 2-D MDW codes with $\mathrm{M}$ as a number of wavelengths, $N$ as a temporary codes length, $W$ as a weight, $\lambda_{a}$ and $\lambda_{c}$ are auto-correlation and crosscorrelation values respectively. There are denoted by $\left(M x N, W, \lambda_{a}, \lambda_{c}\right)$. The $j_{t h}$ user's 2-D codes $C_{M, N}^{j}$, in equation (1), is a matrix of $M$ row vectors $d_{k, N}^{j}$ related to the temporary spreading; $d_{1, N}^{j}=c_{k, 1}^{j}, c_{k, 2}^{j}, \ldots, c_{k, N-1}^{j}, c_{k, N}^{j}$ where $c_{k, i}^{j} \in\{0,1\}$ and $k$ is the emitted wavelength of $k €$ $\{1, \ldots, M\}[5,7,9]$.

$C_{M, N}^{j}=\left[\begin{array}{c}d_{1, N}^{j} \\ d_{2, N}^{j} \\ \cdot \\ \cdot \\ \cdot \\ d_{M-1, N}^{j} \\ d_{M, N}^{j}\end{array}\right]$

The signals $r_{k, N}(t)$ are sum of the temporary spreading data of $\mathrm{Fu}$ user carried on the wavelength $\lambda_{k}$ and expressed as $r_{k, N}(t)=\sum_{j=1}^{F u} b_{i}^{j}(t) d_{k, N}^{j}$, where $b_{i}^{j}(t)$ is $i_{t h}$ user data bit of $j_{t h}$ and $\mathrm{Fu}$ is the number of users. The 
M signals $r_{k, N}^{j}(t)$ are multiplexed and the total signal $R_{M, N}(t)$ transmitted on optical fiber is expressed as $M \times$ $N[5,7,9]$.

$$
R_{M, N}(t)=\left[\begin{array}{c}
r_{1, N(t)} \\
r_{2, N(t)} \\
\cdot \\
\cdot \\
\cdot \\
r_{M-1, N(t)} \\
r_{M, N(t)}
\end{array}\right]
$$

2-D MDW optical CDMA network are made of $M$ (transmitter) and $N$ (receiver). $A_{g, h}$ correspond to the code where $g \in(1,2,3, \ldots, M-1)$ and $h \in$ $(1,2,3, \ldots, N-1) . X_{g}$ and $Y_{k}$ are spectral and spatial encoding respectively. Table 1 shows the 2-D MDW code projections $[5,7,9]$.

Table 1. 2-D MDW Code Projections $k_{1}=4$ and $k_{2}=2$.

\begin{tabular}{|c|c|c|c|}
\hline$X_{g, h} / Y_{g}$ & {$[000011011]$} & {$[011000110]$} & {$[110110000]$} \\
\hline$\left[\begin{array}{l}0 \\
1 \\
1\end{array}\right]$ & {$\left[\begin{array}{l}000000000 \\
000011011 \\
000011011\end{array}\right]$} & {$\left[\begin{array}{l}000000000 \\
011000110 \\
011000110\end{array}\right]$} & {$\left[\begin{array}{l}000000000 \\
110110000 \\
110110000\end{array}\right]$} \\
{$\left[\begin{array}{l}1 \\
1 \\
0\end{array}\right]$} & {$\left[\begin{array}{l}000011011 \\
000011011 \\
000000000\end{array}\right]$} & {$\left[\begin{array}{l}011000110 \\
011000110 \\
000000000\end{array}\right]$} & {$\left[\begin{array}{l}110110000 \\
110110000 \\
000000000\end{array}\right]$} \\
\hline
\end{tabular}

$X=\left[x_{0}, x_{1}, x_{2}, \ldots, x_{M-1}\right]$ and $Y=\left[y_{0}, y_{1}, y_{2}, \ldots, y_{N-1}\right]$ are the code sequence of 1-D MDW codes. The 2-D MDW cross-correlation can be derived through four characteristic matrices of $A^{(d)}$, where $d \in(0,1,2,3)$ are defined as:

$A^{0}=Y^{T} X$

$A^{1}=Y^{T} \bar{X}$

$A^{2}=\bar{Y}^{T} X$

$A^{3}=\bar{Y}^{T} \bar{X}$

The cross-correlation between $A^{(d)}$ and $A_{g, h}$ can be defined as:

$R^{(d)}(g, h)=\sum_{i=0}^{M-1} \sum_{j=0}^{N-1} a_{i j}^{(d)} a_{(i+g)(j+h)}$

Where $a_{i j}^{(d)}$ is $(i, j)_{t h}$ of $A^{(d)}$ and $a_{(i+g)(j+h)}$ is $(i, j)_{t h}$ of $A_{g, h} \quad ; \quad g \in(1,2,3, \ldots, M-1) \quad$ and $h \in$ $(1,2,3, \ldots, N-1)$.

Table 2 shows the cross-correlation of the 2-D MDW code generated from $R^{(d)}(g, h)$. From the table, $R^{(3)}(g, h)$ has nonzero value when $g \neq 0 \cap h \neq 0$. Furthermore, when $g \neq 0$ and $h \neq 0$, the values of $R^{(0)}(g, h), R^{(1)}(g, h), R^{(2)}(g, h)$ and $R^{(3)}(g, h)$ indicates the specific relationships. Cross-correlation function is defined by using $R^{(3)}(g, h)$ to eliminate influence due to $A_{g, h}$ from $R^{(0)}(g, h), R^{(1)}(g, h)$, and $R^{(2)}(g, h)$ when $\neq 0 \cap h \neq 0$. New derived 2-D MDW cross-correlation function is expressed as;
Table 2. 2-D MDW Cross-Correlation.

\begin{tabular}{|c|c|c|c|c|}
\hline$X_{g, h}$ & $R^{(0)}(g, h)$ & $R^{(1)}(g, h)$ & $R^{(2)}(g, h)$ & $R^{(3)}(g, h)$ \\
\hline $\begin{array}{c}g=0, \\
h=0\end{array}$ & $k_{1} k_{2}$ & 0 & 0 & 0 \\
\hline $\begin{array}{c}g=0, \\
h \neq 0\end{array}$ & $k_{1}$ & $k_{1}$ & 0 & 0 \\
\hline $\begin{array}{c}g \neq 0, \\
h=0\end{array}$ & $k_{2}$ & 0 & $k_{2}\left(k_{1}-1\right)$ & 0 \\
\hline $\begin{array}{c}g \neq 0, \\
h \neq 0\end{array}$ & 1 & 1 & $k_{1}-1$ & $k_{1}-1$ \\
\hline
\end{tabular}

$R^{(0)}(g, h)-R^{(1)}(g, h)-\frac{R^{(2)}(g, h)}{\left(k_{1}-1\right)}+\frac{R^{(3)}(g, h)}{\left(k_{1}-1\right)}=$ $\left\{k_{1} k_{2}\right.$, for $g=0$ and $h=1$

0 , otherwise

\section{2-D Wavelength/Time (W/T) Signal-to- Noise Analysis}

Phase-induced intensity noise (PIIN), shot noise and thermal noise are three types of noises that have to be considered in the performance analysis. To identify the thermal lights produces, the photodiode can be expressed as follows [9]:

$<i^{2}>2 e I B+I^{2} B \tau_{c}+\frac{4 K_{b} T_{n} B}{R_{L}}$

The noise power of $I_{P I I N}^{2}$ can be expressed as below.

$\left\langle I_{P I I N}^{2}\right\rangle=\frac{B_{r} \Re^{2} P_{S r}^{2}}{M \Delta f k_{2}^{2}(M N-1)^{2}}\left[\begin{array}{c}k_{1} k_{2}(M N-1)^{2}+ \\ {\left[k_{2}(W-1)(M-1)\right]^{2}}\end{array}\right]$

The short noise current can be written as follow:

$$
\begin{aligned}
& \left\langle I_{\text {short }}^{2}\right\rangle=2 e B_{r}\left\{\frac { \Re ^ { 2 } P _ { S r } ^ { 2 } } { M k _ { 2 } ( M N - 1 ) } \left[k_{1} k_{2}(M N-1)+\right.\right. \\
& 2 k_{1}(W-1)(N-1)+2 k_{2}(W-1)(M-1)+ \\
& \quad 4(W-1)(M-1)(N-1)]\}
\end{aligned}
$$

The thermal noise power is:

$\left\langle I_{\text {thermal }}^{2}\right\rangle=\frac{4 K_{b} T_{n} B_{r}}{R_{L}}$

Total photocurrent output from receiver $I_{r}$ :

$\left\langle I_{r}^{2}\right\rangle=\left[\frac{\Re P_{s r} k_{1}}{M}\right]^{2}$

The signal-to-noise ratio (SNR) equation at receiver is developed as follows:

$S N R=\frac{I_{r}^{2}}{\left\langle I_{\text {PIIN }}^{2}\right\rangle+\left\langle I_{\text {short }}^{2}\right\rangle+\left\langle I_{\text {thermal }}^{2}\right\rangle}$

Therefore, bit error rate (BER) can be expressed in terms of the signal-to-noise ratio (SNR) [9]:

$B E R(M)=\frac{1}{2} \operatorname{erfc}\left(\sqrt{\frac{S N R}{8}}\right)$

where $\operatorname{erfc}$ is: 


$$
\operatorname{erfc}(x)=\frac{2}{\sqrt{x}} \int_{x}^{\infty} e^{\left(-z^{2}\right)} d z
$$

\section{Simulation Results and Discussions}

Table 3. Table of link parameters.

\begin{tabular}{|l|l|}
\hline \multicolumn{2}{|c|}{ Parameters Used in Numerical Calculation } \\
\hline $\begin{array}{l}\text { PD quantum efficiency } \\
\text { Spectral width of } \\
\text { broadband light source }\end{array}$ & $\eta=0.6$ \\
Operating wavelength & $\Delta \lambda=30 \mathrm{~nm}(\Delta=3.75 \mathrm{THz})$ \\
Electrical bandwidth & $\lambda_{0}=1.55 \mu \mathrm{m}$ \\
Data transmission rate & $\mathrm{B}=311 \mathrm{MHz}$ \\
Receiver noise temperature & $\mathrm{R}_{\mathrm{b}}=622 \mathrm{Mbps}$ \\
Receiver load resistor & $\mathrm{T}_{\mathrm{L}}=300 \mathrm{~K}$ \\
Boltzmann's constant & $\mathrm{K}_{\mathrm{b}}=1.38 \times 10^{-23} \mathrm{~W} / \mathrm{K} / \mathrm{Hz}$ \\
Electron charge & $e=1.6021764 \times 10^{-19}$ coulombs \\
Light velocity & $\mathrm{C}=3 \times 10^{8} \mathrm{~m} / \mathrm{s}$ \\
\hline
\end{tabular}

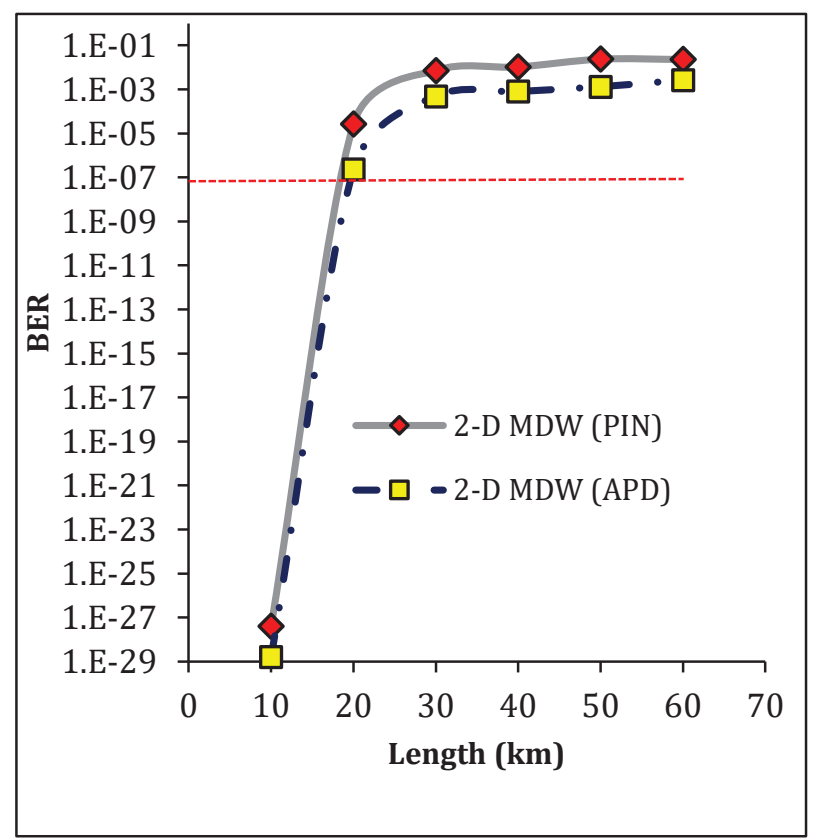

Fig. 1. BER versus optical fiber length for 2-D MDW codes.

Table 3 is the system parameters used to find the numerical results, while Figure 1 demonstrates the graph of BER versus optical fiber length in kilometer $(\mathrm{km})$ for the 2-D MDW codes. In this simulation, $622 \mathrm{Mbps}$ of bit rate is used. The number of length used is from $10 \mathrm{~km}$ until $60 \mathrm{~km}$. According to the simulation and the result obtained, the graph increase for both bit rate due to the increasing of the optical fiber length. The system performances of BER $10^{-9}$ 2-D MDW (APD) at $19 \mathrm{~km}$ of fiber length have better performance compared to 2-D MDW (PIN) at fiber length of $18 \mathrm{~km}$. This is because of the distance described by the number of dispersion and the signal loss.

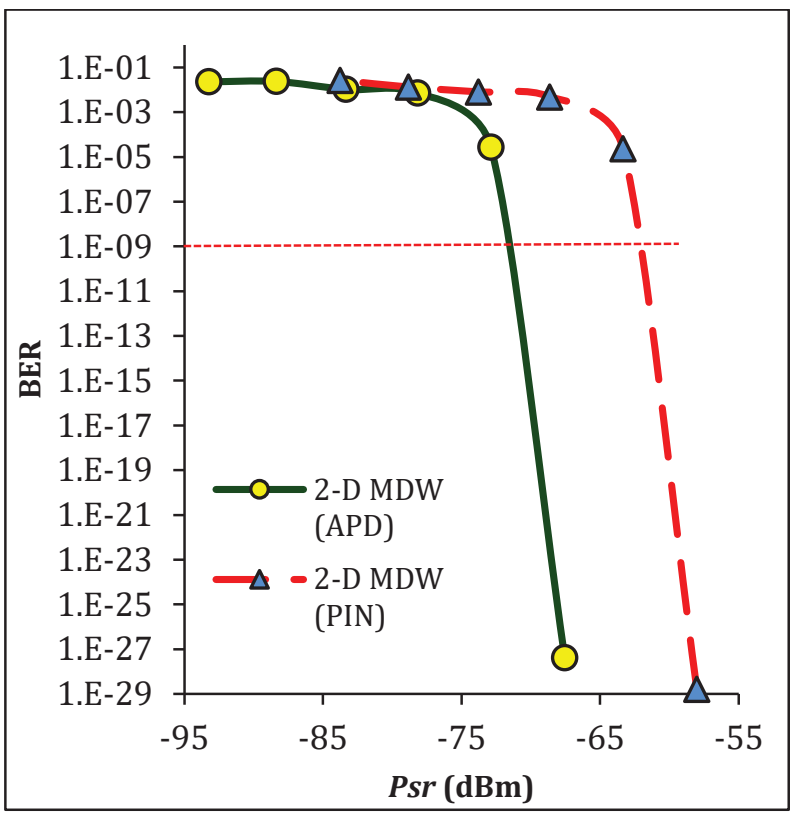

Fig. 2. BER versus effective received power $(P s r)$ for 2-D MDW codes.

Figure 2 illustrates the curve of BER versus effective received power (Psr) for 2-D MDW codes. The values of effective received power $(P s r)$ are varied from $-95 \mathrm{dBm}$ until $-40 \mathrm{dBm}$. The number of bit rate used is $622 \mathrm{Mbps}$. Then the length of optical fiber used is $10 \mathrm{~km}$ to $60 \mathrm{~km}$. It can be seen that, 2-D MDW (APD) code requires $-71 \mathrm{dBm}$ more effective source power to achieve normal optical transmission requirements compared to 2-D MDW (PIN), which requires only $-61 \mathrm{dBm}$. This situation occurs when the losses of fiber optic cable caused the Psr to decrease while the BER is increased.

\section{Conclusion}

From the numerical results, the $P s r$ value for 2-D MDW (APD) codes is $-71 \mathrm{dBm}$ while 2-D MDW (PIN) codes is $-61 \mathrm{dBm}$. Therefore, photodiode APD are more effective compared to photodiode PIN because of photodiode APD are increase according their gain. In addition, the theoretical calculation demonstrates the number of BER $10^{-9}$ so, it's easily to suppress PIIN and eliminate MAI.

The author would like to acknowledge this work was supported by the High Ministry of Education Malaysia under the Fundamental Research Grant Scheme (FRGS) under grant \#9003-00456.

\section{References}

1. C. B. M. Rashidi, S. A. Aljunid, F. Ghani, H. A. Fadhil, M. S. Anuar, A. R. Arief, Optik-International Journal for Light and Electron Optics, 125, 17, pp. 4889-4894 (2014)

2. V. Jyoti, R. S. Kaler, Optik, pp. 851-857 (2011)

3. A. R. Arief Jamil Abdullah, S. A. Aljunid, A. M. Safar, J. M. Nordin, R. B. Ahmad, Fiber Optics and Optical Communication, 51, 6 (2012) 
4. C.C. Yang, Optical Fiber Technology, 14, pp. 134$142(2007)$

5. A. R. Arief, S. A. Aljunid, M. S. Anuar, M. N. Junita, R. B. Ahmad, F. Ghani, 3rd International Conference on Photonics, (2012)

6. C. B. M. Rashidi, S. A. Aljunid, M. S. Anuar, Hilal A. Fadhil, F. Ghani, Journal of Theoritical and Applied Information Technology, 61 (2014)

7. A. R. Arief, S. A. Aljunid, M. S. Anuar, M. N. Junita, R. B. Ahmad, F. Ghani, IEEE International Conference in Control System, Computing and Engineering (ICCSCE), pp. 488-494 (2011)

8. C. C. Yang, IEEE Photonics Technology Letters, 17, 6, pp. 1343-1345, (2005)

9. A. R. Arief, S. A. Aljunid, M. S. Anuar, M. N. Junita, R. B. Ahmad, Optik - International Journal for Light and Electron Optics, pp. 3786-3793 (2013)

10. N. Din Keraf, S. A. Aljunid, A. R. Arief, M. S. Anuar, C. B. M. Rashidi, P. Ehkan, M. N. Nurol, International Conference on Electronic Design (ICED), (2014) 